\title{
TRAFFIC CONTROLLING BY WIRELESS SENSOR NETWORKS
}

\begin{tabular}{|l|l|}
\hline Lata Ganesh Naik & Mrs. Shreedevi \\
MCA, RNS Institute of Technology & Assistant Professor \\
Bengaluru, India & MCA, RNS Institute of Technology \\
latanaik20@gmail.com & Bengaluru, India \\
& sirisuresh2002@gmail.com \\
\hline
\end{tabular}

\begin{abstract}
Vehicular traffic is continuously escalating around the world, especially in large urban areas. The increasing Vehicle inhabitants call for a foremost enhancement and modernization in the presented Traffic Signaling systems in both developing and developed country. The currently used traffic system is a simple time based system. And it works on a instance intermission origin which is now unproductive for haphazard and non homogeneous interchange. The Concept Proposed in this paper involves the use of Wireless sensor network technology to intellect occurrence of interchange in the neighborhood of whichever sphere or crossroads and then capable to itinerary the interchange pedestal on interchange accessibility or by density in preferred track. This scheme does not oblige whichever structure in vehicles therefore it can be put into service in whichever interchange structure to a certain extent effortlessly with less instance and also less expensive. Wireless sensor networks Technology is used on the way to intelligence truck and a microcontroller pedestal steering algorithm which is planned for outstanding Traffic management. Traffic inspection arrangement provide the information used by Intelligent Transportation Systems (ITS).
\end{abstract}

Keywords: Traffic inspection, Vehicular traffic, wireless sensors, Traffic Signaling, Intelligent Transportation Systems (ITS).

\section{Introduction}

The Traffic thickness is expanding in creating nations which require the need of Advance canny Traffic signs to supplant the anticipated manual and time based Traffic sign framework. Exploratory framework in survival include picture handling based thickness recognizable proof for steering of activity which may be inadequate in circumstance like mist, rain and dust and so forth. The framework proposed here includes confined Traffic steering for every convergence intersection in view of remote sensor Networks. The proposed framework has a deepest microcontroller at each intersection which gets information from little remote sensor hubs put on the Road. The sensor hubs have sensors that can identify the vicinity of vehicle and the transmitter remotely transmits the movement thickness to the focal programmable microcontroller. The Microcontroller makes utilization of the proposed customized calculation to discover approaches to oversee and control activity in a productive system effectively.

\section{Literature survey}

Wireless sensor networks (WSNs) have seen express development because of the extraordinary advancement in microelectronics and electromechanical frameworks. The current techniques for movement administration, review and oversee are not adequate as far as the execution, cost, and the exertion required for upkeep and backing. To stay away from the sticking issue the U.S. government starts the Federal Intelligent Transportation System (ITS) project in 1991 for the development and arrangement of cutting edge innovations for boosting the movement limit and minimizing the postponement.

Most traditional activity observation frameworks use meddling sensors, which incorporate inductive circle finders, smaller scale circle tests, pneumatic street tubes, piezoelectric links and other say something movement sensors. They are picked in view of their high exactness for vehicle discovery (which is $>97 \%$ ). For boosting the benefit from every one of these ITS advancements, there must be a substantial scale arrangement of movement controls on every single boss interstate and neighborhood avenues. Hence, ongoing movement data at all these destinations is needed. Remote sensor systems were created and executed as an activity observation framework with recognition precision on a par with that of inductive circle indicators. They offer an exceptionally alluring different option for inductive circles for activity observation.

The sensor systems have much higher setup agility, which define the framework extendable and deployable everywhere in the exchange system. Remote sensor system assembles the entry data and controls the trade stream as indicated by the arriving exchange information. An insightful movement shine framework detects the event or nonexistence of engine vehicles then it controls the exchange enlightenment subsequently. The motivation in the back speedy trade structures is that drivers won't waste pointless time sitting tight for the movement light to change which may escort them to a few exchange infringement and mishap when a few drivers start to be crushed their resistance.

\section{Architecture}

The remote sensor systems advances can be named meddlesome, non-nosy and off-roadway innovations. Meddlesome activity sensors are introduced inside or over the asphalt. Nonintrusive sensors can be introduced above or in favor of streets with least interruption to movement stream. Off-roadway advancements needn't bother with any particular hardware to be introduced at the test site. In this area, a few normal advancements in each of these classes are contemplated.

\section{Intrusive Technologies}

Intrusive technologies refer to those that oblige establishment specifically onto the asphalts, in saw-cut, openings or burrowing 


\section{Asia Pacific Journals}

under the surfaces. Disadvantages incorporate the disturbance of movement for establishment and repair, disappointments affected by poor street conditions, and framework reinstallation brought about by street repairs or reemerges. example contain inductive disk pneumatic thoroughfare hose, piezoelectric lead, and weigh-inmotion coordination.

\subsection{Inductive Loop detector}

Inductive Loop detectors are the most normally utilized sort of vehicle recognition in view of their dependability in reporting vehicle vicinity. Be that as it may, circle finder establishment can be costly as a result of the physical association needed to interface the circles back to the activity bureau. Such associations are at times infeasible at areas, for example, extensions and inclines. Besides, saw-cut inductive circles are especially touchy to dampness and wire breaks connected with asphalt disappointment.

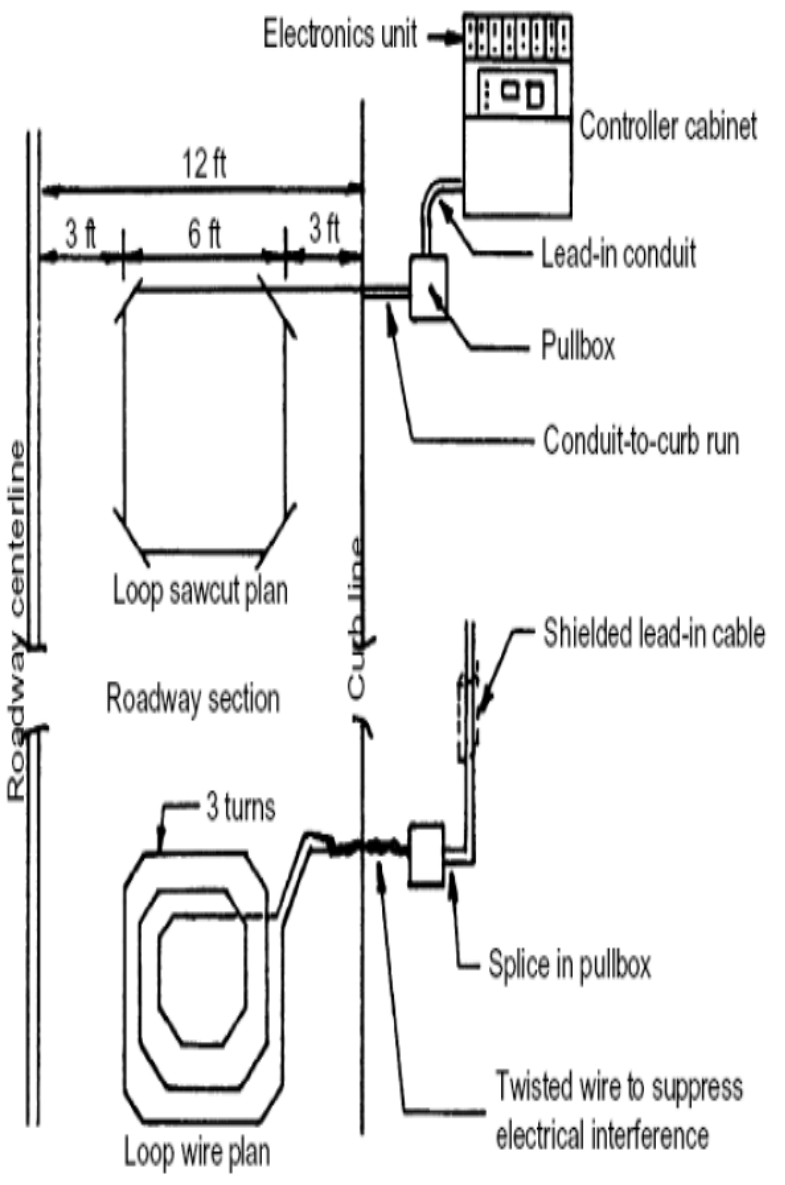

$1 \mathrm{ft}=0.305 \mathrm{~m}$

Fig 1.1: Inductive Loop detector

\subsection{Piezoelectric Sensor}

Piezoelectric sensor is introduced by implanting it under the asphalt. It is built by an uncommonly prepared material (quartz) that will create a 15 voltage when subjected to mechanical effect or vibration. The voltage extent is corresponding to the power or heaviness of the motor vehicle. Since the electrical energy is just produced when the connected power is changing, the estimation will rot to zero if the vehicle remains focused sensor. Other than vehicle identification, grouping is finished by hub tally, separating and weight. In light of its capacity of weight estimation, it is regularly utilized as a component of a say something movement framework. Its downsides are like those of inductive circle, including interruption of activity for establishment and repair, disappointments brought about by movement stress and reemerging, and affectability reliance on temperature and vehicle speed.

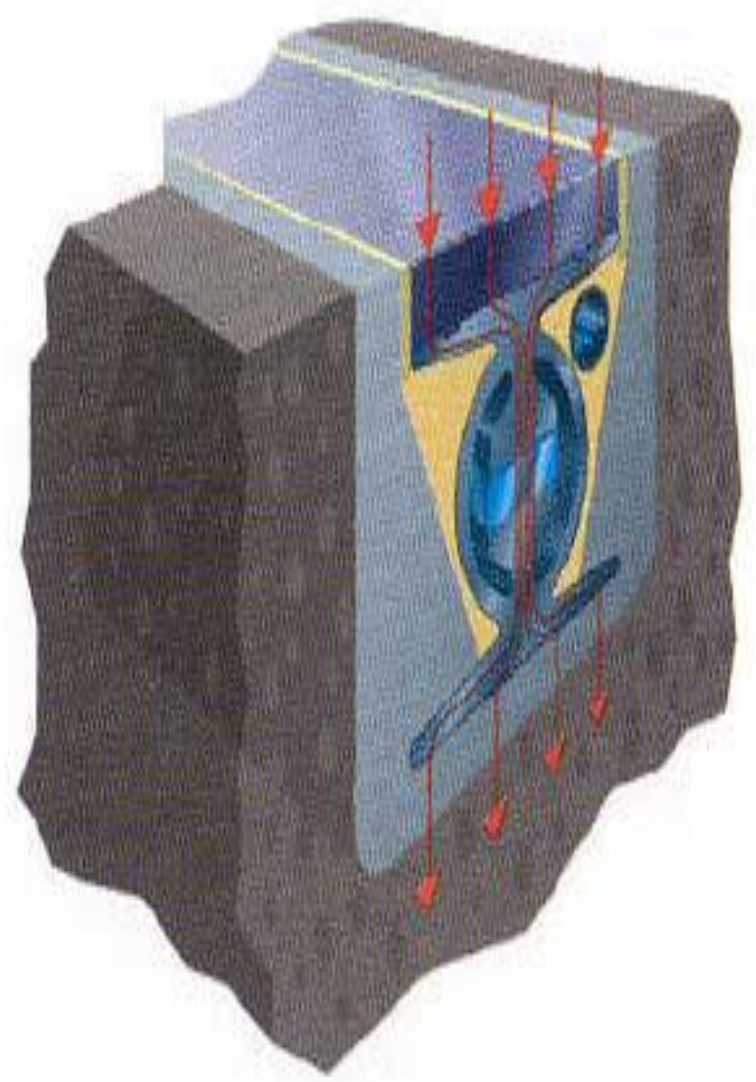

Fig 1.2: Piezoelectric Sensor

\section{Non-Intrusive Technologies}

Non-intrusive technologies needn't bother with any establishment on or under the asphalt, so that the establishment and repair of such a framework should be possible without upsetting the movement. The indicators are typically set of connections on the pavement, or at an visual projection position. Cases of this sort of innovation incorporate microwave radar, infrared, Video Image Processing (VIP), ultrasonic and passive acoustic detectors.

\subsection{Video Imaging Vehicle Detection Systems (VIVDS)}

Video system includes one or several video cameras, microprocessorbased equipment for digitizing and dispensation the descriptions, computer and software for examine the images to take out traffic information. Video Imaging Vehicle Detection Systems (VIVDS) have beaten a percentage of the issues with circles, for example, 


\section{Asia Pacific Journals}

movement interruption and asphalt debasement; yet they have not been as exact in all climate and light conditions as initially expected.

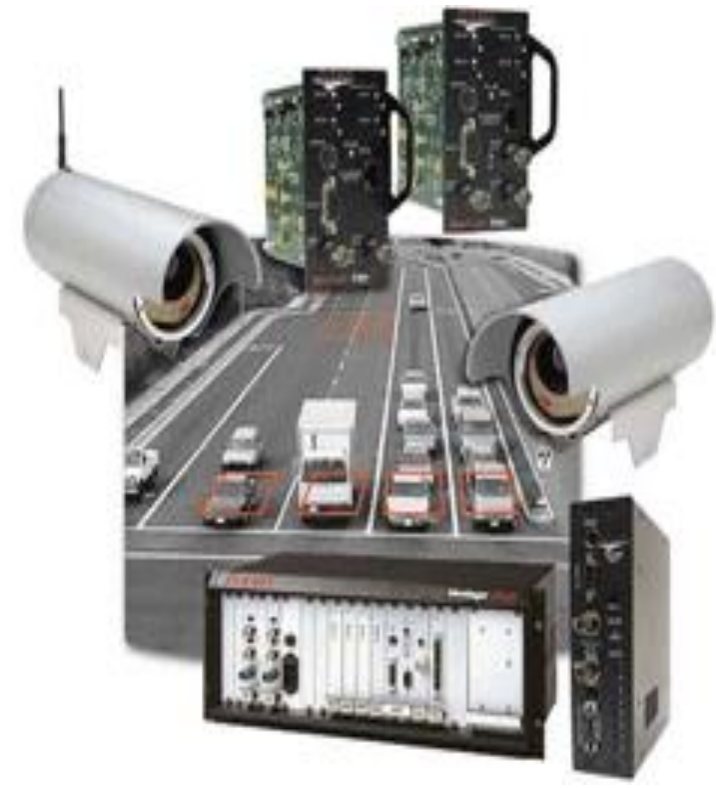

Fig 2.1: Video Imaging Vehicle Detection Systems

\subsection{Microwave radar system}

Microwave Radar system innovation to measure locality and pace of drawing nearer vehicles in certain separation extent in light of the sensor highlights. It is all around tried and exceptionally effective.

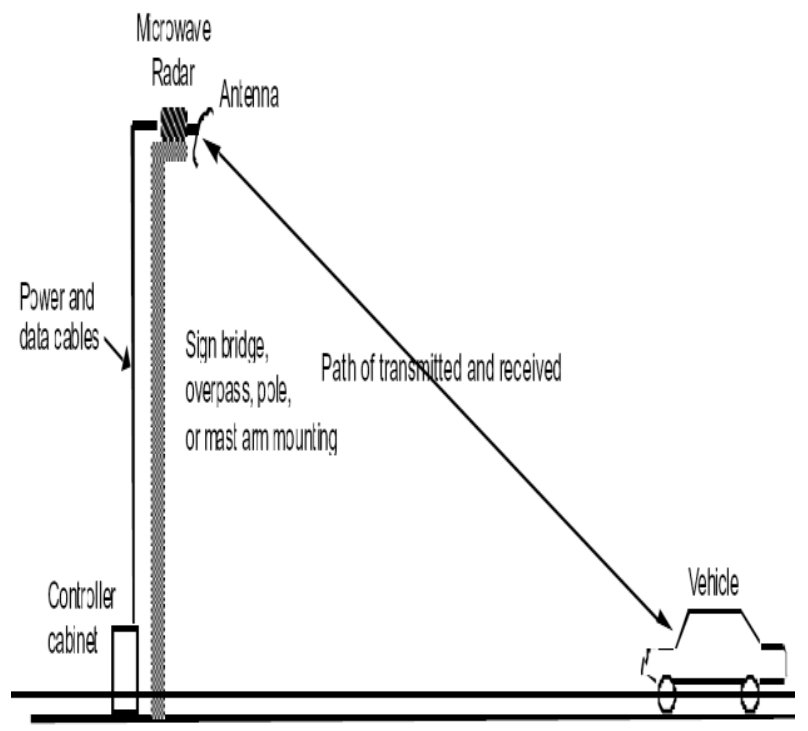

Fig 2.2: Microwave radar system

\subsection{Ultrasonic Detectors}

Transmit sound at $25 \mathrm{KHz}$ to $50 \mathrm{KHz}$ (contingent upon the maker). These frequencies lie over the capable of being heard area. A part of the transmitted vitality is reflected from the street or vehicle surface into the recipient bit of the instrument and is prepared to give vehicle section and vicinity. A run of the mill ultrasonic vicinity identifier transmits ultrasonic vitality as heartbeats.

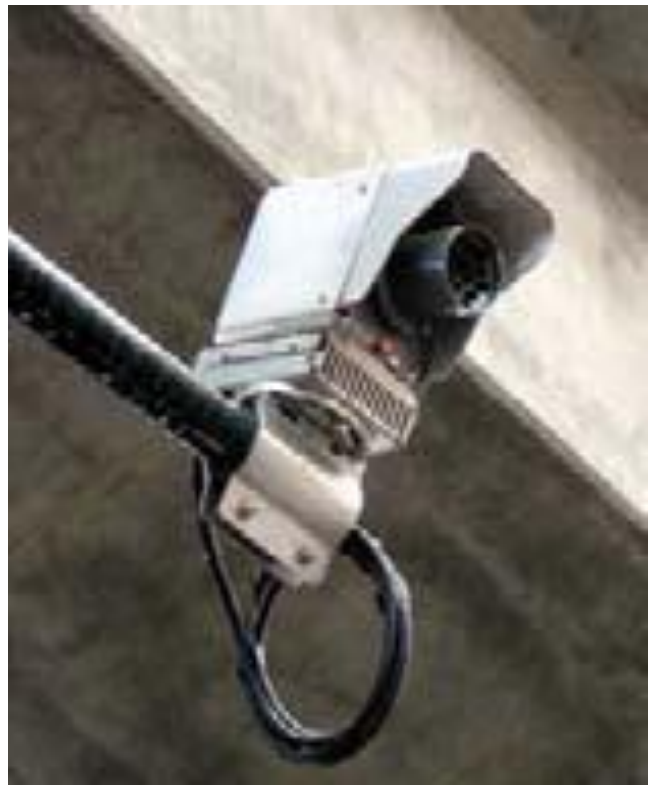

Fig 2.3: Ultrasonic Detectors

\subsection{Infrared vehicle detection system}

Infrared (IR) radiation is electromagnetic radiation with wavelength greater than that of observable radiance but shorter than that of radio influence. Frequent methods for traffic inspection use IR assorting from 100 to $105 \mathrm{GHz}$. There are two types of IR-based system, active and passive.

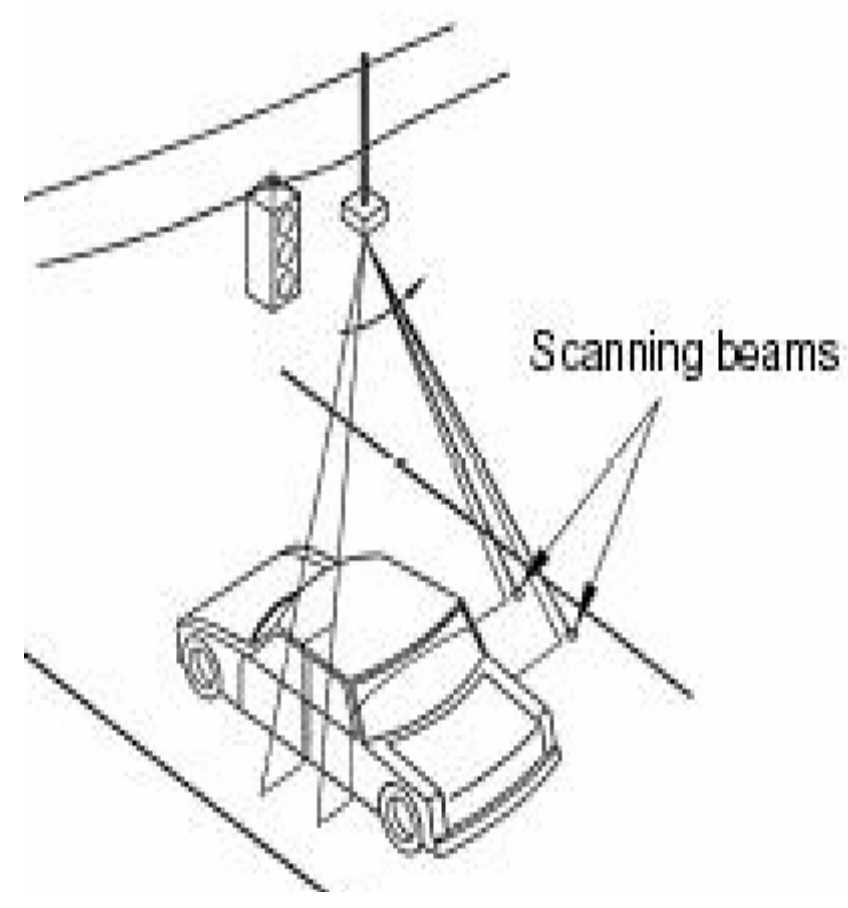

Fig 2.4: Infrared vehicle detection system

\subsection{Passive acoustic detectors.}




\section{Asia Pacific Journals}

Vehicular movement produces acoustic vitality or capable of being heard sound from a mixed bag of sources inside of the vehicle and from the connection of the vehicle's tires with the street surface. Varieties of acoustic receivers are utilized to pick-up these sounds from an engaged range inside of a path on a roadway. At the point when a vehicle goes through the location zone, the sign handling calculation distinguishes an increment in solid vitality and a vehicle vicinity sign is created. At the point when the vehicle leaves the recognition zone, the sound vitality diminishes beneath the identification limit and the vehicle vicinity sign is ended.

\section{Off-Roadway Technologies}

Off-Roadway Technologies refer to those that needn't bother with any equipment to be setup under the asphalt or on the roadside. It incorporates test vehicle advances with Global Positioning System (GPS) and cell telephones; Automatic Vehicle Identification (AVI); and remote detecting advances that make utilization of pictures from flying machine or satellite.

\subsection{Probe Vehicles with Global Positioning System (GPS)}

GPS is a satellite course-plotting scheme initially residential by the United States Department of resistance, legitimately named NAVSTAR GPS in 1978 [2.22]. A group of more than 24 GPS satellites transmit perfect moment in time radio gesture to GPS beneficiary. The location and speed is calculated by the procedure that correctly computes the time difference of arrival (TDOA) of a signal transmits from three or more coordinated transmitters. The structure is accessible for open uses in civilian submission as a public good.

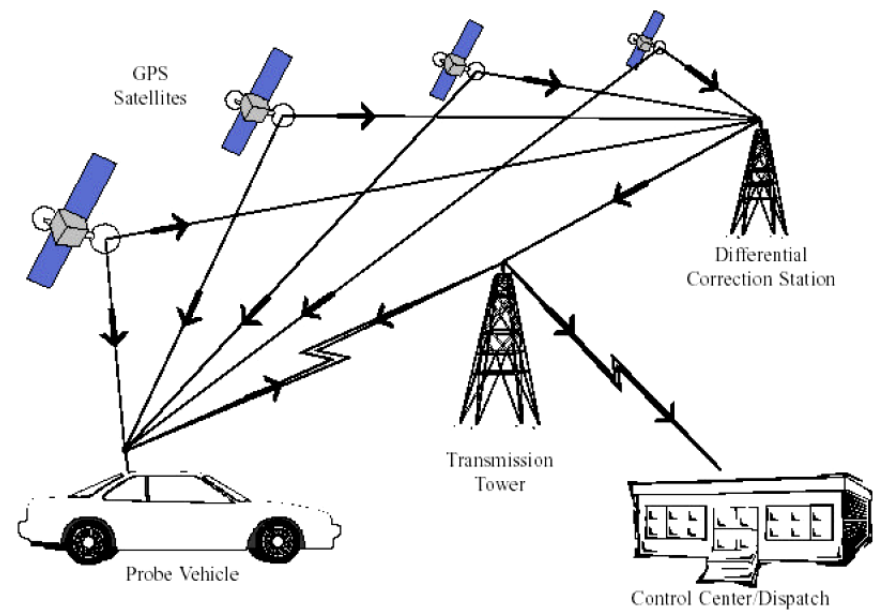

Fig 3.1: Sample configuration of a GPS-based probe vehicle system

\section{References}

[1]. Rashid Hussian, Sandhya Sharma,Vinita Sharma and Sandhya Sharma, "WSN Applications: Automated Intelligent Traffic Control System Using Sensors," in IJSCE ' 3, July 2013, ISSN: 2231-2307. [2]. Faisal Ahmed Al-Nasser and Magdi S. Mahmoud, "Wireless Sensors Network Application: A Decentralized Approach for Traffic Control and Management".

[3]. Khalil M. Yousef, Jamal N. AL-Karaki and Ali M. Shatnawi, "Intelligent Traffic Light Flow Control System Using Wireless Sensors Networks," 2010.
[4]. Sing Yiu Cheung, Sinem Coleri, Ram Rajagopal and Pravin Varaiya, "A wireless sensor network for traffic surveillance". [5]. Sing-Yiu Cheung Pravin Varaiya, "Traffic Surveillance by Wireless Sensor Networks: Final Report,” in January 29, 2007.

\section{Conclusion}

The proposed paper covers the range of remote sensors system and signalized movement control, which depends emphatically on these gadgets. A few sorts of identifiers were recorded with some data about distinctive sort. A versatile activity sign time control calculation in light of another movement foundation utilizing WSNs is proposed on a solitary and various street convergences. The framework is self-arranging and works progressively to distinguish movement states and trade data with different hubs by means of a remote correspondence with self-recuperation capacity. What's more, no movement disturbance will be essential when Another activity sensor is to be introduced. 